\title{
Teor de sódio em preparações oferecidas por restaurantes comerciais da cidade de Itaqui, RS
}

\author{
Carla Adriana Thones Machado Borges ${ }^{1}$ e Tiago André Kaminski ${ }^{2}$
}

O trabalho se propôs a avaliar teor de sódio em preparações oferecidas por restaurantes comerciais do tipo selfservice da cidade de Itaqui. Foram realizadas três coletas de alimentos cozidos (arroz branco, feijão em caldo e carne assada) em cinco restaurantes. Previamente à análise de sódio por fotometria de chama, as amostras foram submetidas à pré-secagem, moagem e digestão nitroperclórica. Os resultados obtidos evidenciam a falta de padronização no uso de sal devido à grande variação no teor de sódio entre restaurantes e entre as amostras coletadas em um mesmo restaurante. Os teores de sódio encontrados em cerca de $90 \%$ dos alimentos avaliados superaram as recomendações dietéticas. Com base nas recomendações do Programa de Alimentação do Trabalhador, a somatória das porções de arroz, feijão e carne nas quantidades estabelecidas pelo Guia Alimentar só não superou o limite máximo de sódio para a refeição almoço em uma coleta de um dos restaurantes. Conclui-se que o sal é demasiadamente utilizado nas preparações analisadas dos restaurantes, estando em conformidade com recomendações dietéticas apenas em duas amostras de feijão (15\%) e três de carne (20\%).

Palavras-chave: Sal; Hipertensão; Alimentos; Alimentação fora do lar.

\section{Sodium content in preparations offered by commercial restaurants in Itaqui, Rio Grande do Sul, Brazil}

The aim of this work was to evaluate sodium content in preparations offered by commercial self-service restaurants in the Itaqui city. Three collects of cooked food (white rice, bean in broth and roast meat) were performed in five restaurants. The samples were submitted to pre-drying, milling and nitropercloric digestion previously to the sodium assessment through flame photometry. The results obtained evidenced the lack of standardization in the use of salt due to the great variation in the sodium content between restaurants and among the samples collected from the same restaurant. The sodium contents found in $90 \%$ of the foods evaluated exceeded dietary recommendations. Based on the recommendations of the Workers' Food Program, the sum of the portions of rice, beans and meat in the quantities established in the Brazilian Food Guide did not exceed the maximum sodium limit for the lunch meal in one collect of one of the restaurants. It is concluded that the salt is overused in the food preparations analyzed, complying with dietary recommendations only in two samples of beans $(15 \%)$ and three samples of meat $(20 \%)$.

Keywords: Salt; Hypertension; Foods; Feeding outside home.

\footnotetext{
${ }^{1}$ Nutricionista, Universidade Federal do Pampa (UNIPAMPA). E-mail: carla_thones_borges@hotmail.com

2 Professor Doutor, Universidade Federal do Pampa (UNIPAMPA). Endereço para correspondência: Universidade Federal do Pampa, Rua Luiz Joaquim de Sá Britto, s/n, Bairro Promorar, Itaqui, Rio Grande do Sul. CEP: 97650-000. Telefone: +55 (55) 99907 0662. E-mail: tiagokaminski@unipampa.edu.br
} 


\section{INTRODUÇÃO}

O sódio é um íon encontrado naturalmente em grande parte nos alimentos e como parte do sal de cozinha na forma de cloreto de sódio. A parcela de sódio que ocorre naturalmente nos alimentos é geralmente inferior à parcela adicionada, na forma de sal ou aditivos, por produtores, manipuladores de alimentos e consumidores ${ }^{[1]}$.

O cloreto de sódio é um produto bastante apreciado na gastronomia, sendo rotineiramente adicionado no preparo dos alimentos no intuito de melhorar o sabor e conferir propriedades de conservação [2].

O sódio é um nutriente essencial que contribui na regulação osmótica dos fluidos, atua na condução de estímulos nervosos e na contração muscular [1]. No entanto, o consumo em excesso desse nutriente é associado ao desenvolvimento de Doenças Crônicas Não Transmissíveis (DCNT), que estão entre as primeiras causas de internações e óbitos no mundo [1]. Apesar da redução nas taxas de mortalidade por DCNT, a Hipertensão Arterial Sistêmica (HAS), que é associada à origem de muitas DCNT, ainda é de alta prevalência, inclusive em crianças e adolescentes, indicando um fator prognóstico para a HAS na idade adulta ${ }^{[3,4]}$.

O consumo de sal, na maioria dos países, é excessivo, variando de 9 a $12 \mathrm{~g}$ por pessoa por dia [5]. Spinelli et al. ${ }^{[6]}$ estimaram que o consumo médio de sal da população brasileira seja de 9,6 $\mathrm{g} / \mathrm{pessoa} / \mathrm{dia}$, enquanto Salas et al. [7] classificaram o Brasil entre os maiores consumidores mundiais de sal, com média de ingestão de 15,09 $\mathrm{g} / \mathrm{pessoa} /$ dia. Já Sarno et al. ${ }^{[8]}$ estimaram a disponibilidade de sódio domiciliar no Brasil, a partir dos dados da Pesquisa de Orçamentos Familiares (POF), em 4,7 $\mathrm{g} / \mathrm{pessoa} /$ dia. Em contraste, a Organização Mundial da Saúde (OMS) [9] recomenda uma ingestão diária de, no máximo, $5 \mathrm{~g}$ de sal (equivalentes a $2 \mathrm{~g}$ de sódio) para adultos.Paralelamente, de acordo com a POF 2008-2009, houve um aumento de 7\% do gasto com alimentação fora de casa, pois o percentual de despesas monetárias e não monetárias nesta categoria era de $24,1 \%$ na POF 2002-2003 e passou para $31,1 \%$ [10]. Este dado é mais relevante, se considerado que aproximadamente $80 \%$ do consumo de sódio pelos indivíduos provêm de alimentação fora de casa e de alimentos prontos para o consumo [11].
Das questões que envolvem a alimentação fora de lar, destacam-se as questões espaciais e temporais, pois os indivíduos não têm tempo suficiente para preparar e/ou realizar suas refeições em casa [12]. O setor de alimentação fora de casa engloba tanto estabelecimentos coletivos quanto comerciais [13]. Nessas categorias, o restaurante por peso no modelo self-service é muito frequentado pelos brasileiros, no qual o comensal escolhe o que deseja consumir e paga o valor referente ao peso dos alimentos colocados em seu prato [14]. Indivíduos jovens residentes em domicílios situados na área urbana e em regiões economicamente mais desenvolvidas, como Sul e Sudeste, têm maior frequência de alimentação fora do lar [15].

A cidade de Itaqui, na região da Fronteira Oeste do Rio Grande do Sul, tem uma população de 38.159 habitantes [16] que tem passado por mudanças de hábitos alimentares, constatadas principalmente pelo aumento do número de restaurantes no município.

Diferente dos restaurantes de alimentação coletiva, nos restaurantes comerciais não há exigência legal de um responsável técnico nutricionista [17]. Nesse caso, a tarefa de elaborar receitas é, geralmente, delegada ao cozinheiro, que pode fazer uso do sal e diversos temperos, com alto teor de sódio, para obter incremento no sabor dos alimentos e agradar ao paladar dos clientes, que podem vir a consumir alimentos não saudáveis e desequilibrados em nutrientes ${ }^{[6]}$.

As evidências, que relacionam o consumo excessivo de sódio com o maior risco de desenvolver enfermidades crônicas, são suficientes para justificar a redução do teor de sal nas preparações de restaurantes [6]. Porém, há certa carência de publicações sobre a incidência de sódio em alimentos servidos por restaurantes, principalmente quando a pesquisa envolve restaurantes comerciais. Os trabalhos que avaliaram os teores de sódio em preparações produzidas por restaurantes demonstraram a elevada incidência deste elemento [6,7,18,19,20], o que faz com que a quantidade diária máxima recomendada pela OMS seja facilmente superada.

Neste contexto, o trabalho foi realizado com o objetivo de avaliar o teor de sódio em preparações habitualmente oferecidas em 
restaurantes comerciais do tipo self-serviceda cidade de Itaqui, RS,demonstrando a incidência de sódio por tipo de alimento, estabelecimento, coleta e em relação às recomendações do Guia Alimentar para a População Brasileira.

\section{METODOLOGIA}

Amostras: foram coletadas amostras de três alimentos cozidos (arroz branco, feijão em caldo e carne bovina) servidos em cinco restaurantes comerciais do tipo self-service (identificados como 1, 2, 3, 4 e 5) localizados na região central da cidade de Itaqui, RS. Os restaurantes foram escolhidos por conveniência, a partir da aceitação dos responsáveis pelo estabelecimento através de um termo de autorização, que representou o interesse em participar da pesquisa e a permissão para a coleta das amostras. Previamente à assinatura do termo de autorização, os responsáveis pelos estabelecimentos foram esclarecidos dos objetivos do trabalho e receberam um termo de confidencialidade, como garantiade anonimato do estabelecimento e do retorno dos resultados, porém não foram informados dos dias de coleta das amostras. Foram realizadas três coletas em diferentes dias dos meses de junho e julho de 2014, onde aproximadamente $300 \mathrm{~g}$ das amostras oferecidas no buffet foram transferidas para pratos de alumínio, fechadas e imediatamente encaminhadas ao Laboratório de Química da Unipampa - campus Itaqui.

Preparo das amostras: as preparaçõesforam submetidas à pré-secagem em estufa com circulação de ar (SL 102/480, Solab) a $55^{\circ} \mathrm{C}$ por 24 horas. Posteriormente, as amostras parcialmente secas foram moídas em micromoinho (A11, IKA), embaladas em sacos de polietileno, fechadas e mantidas sob congelamento à $-18^{\circ} \mathrm{C}$ até etapa de mineralização.

Análise de sódio: em bloco digestor (NT 352, Novatecnica), as amostras foram mineralizadas através de digestão nitroperclórica [21] e analisadas por espectrometria de absorção atômica, modalidade chama (DM-62, Digimed), com comprimento de onda de $589 \mathrm{~nm}$ e prévia calibração com padrões de 0 e 20 mg de sódio.L-1 ${ }^{-1}$.

Tratamento dos dados: as repetições das amostras foram analisadas em triplicata, os resultados submetidos à análise de variância e as médias comparadas pelo teste de Tukey em nível de $5 \%$ de significância no programa Statistica, versão 8.0 [22]. Os resultados foram expressos $\mathrm{em} \mathrm{mg}$ de sódio para $100 \mathrm{~g}$ de cada alimento cozido e para as porções dos alimentos cozidos (125 g de arroz, $86 \mathrm{~g}$ de feijão e $90 \mathrm{~g}$ de carne bovina) estabelecidas no Guia Alimentar para a População Brasileira [23].

\section{RESULTADOS}

Conforme resultados descritos na Tabela 1, os teores de sódio foram superiores nas amostras de arroz coletadas no restaurante 2, embora sem diferença significativa do restaurante 4 na $1^{\text {a }}$ coleta; enquanto que o restaurante 3 apresentou os menores teores de sódio no arroz nas três coletas e na média das coletas. Também foram observadas diferenças significativas entre as coletas que, com exceção do restaurante 1, apresentaram maiores teores de sódio na $3^{\text {a }}$ coleta.

Tabela 1. Teor de sódio no arroz branco cozido oferecido pelos restaurantes comerciais do tipo self-service. Itaqui, RS, 2014

\begin{tabular}{c|cccc}
\hline \multirow{2}{*}{ Restaurante } & 1a coleta & 2a coleta & 3a coleta & Médias \\
\cline { 2 - 5 } & \multicolumn{4}{|c}{ mg por 100 g de alimento } \\
\hline $\mathbf{1}$ & $505,1 \pm 8,2^{\mathrm{B} \mathrm{a}}$ & $377,4 \pm 9,0^{\mathrm{Cb}}$ & $336,8 \pm 6,6^{\mathrm{D}}$ & $406,4 \pm 76,4^{\mathrm{C}}$ \\
$\mathbf{2}$ & $544,8 \pm 14,8^{\mathrm{AB}}$ & $679,7 \pm 19,0^{\mathrm{Ab}}$ & $788,7 \pm 33,2^{\mathrm{Aa}}$ & $671,0 \pm 107,8^{\mathrm{A}}$ \\
$\mathbf{3}$ & $277,3 \pm 3,6^{\mathrm{Da}}$ & $174,4 \pm 9,1^{\mathrm{D} \mathrm{b}}$ & $283,4 \pm 18,7^{\mathrm{Da}}$ & $245,0 \pm 54,1^{\mathrm{D}}$ \\
$\mathbf{4}$ & $560,4 \pm 30,2^{\mathrm{A} \mathrm{b}}$ & $440,6 \pm 20,6^{\mathrm{B}}$ & $645,6 \pm 20,1^{\mathrm{B} \mathrm{a}}$ & $548,9 \pm 91,6^{\mathrm{B}}$ \\
$\mathbf{5}$ & $350,1 \pm 18,9^{\mathrm{Cc}}$ & $458,1 \pm 9,1^{\mathrm{B} \mathrm{b}}$ & $558,1 \pm 52,4^{\mathrm{Ca}}$ & $455,4 \pm 94,4^{\mathrm{BC}}$ \\
\hline
\end{tabular}

Valores expressos como média \pm desvio padrão seguidos por letras que indicam diferença estatística significativa em nível de 5\% pelo teste de Tukey; letras maiúsculas correspondem às diferenças estatísticas entre as médias das colunas e letras minúsculas às diferenças entre as médias das linhas 
$\mathrm{Na}$ Tabela 2 está demonstrado o teor de sódio no feijão cozido, sendo que o restaurante 4 apresentou maiores teores de sódio para o feijão cozido em todas as coletas e, consequentemente, na média das coletas; enquanto que o restaurante 5 teve menores teores na $1^{\mathrm{a}}$ e $2^{\mathrm{a}}$ coletas, e $\mathrm{o}$ restaurante 1 na $3^{\text {a }}$ coleta. Assim como no arroz (Tabela 1), os maiores teores de sódio ocorreram na $3^{a}$ coleta, com exceção da amostra de feijão cozido do restaurante 2 , que teve menor incidência de sódio na $3^{\text {a }}$ coleta do que nas anteriores.De modo geral, as médias das coletas demonstram que maiores teores de sódio estiveram nas amostras de feijão dos restaurantes 2 e 4, enquanto que as amostras dos restaurantes 1,2 e 3 tiveram menores teores de sódio.

Tabela 2. Teor de sódio no feijão em caldo cozido oferecido pelos restaurantes comerciais do tipo self-service. Itaqui, RS, 2014

\begin{tabular}{c|cccc}
\hline \multirow{2}{*}{ Restaurante } & 1a coleta $^{\text {a }}$ & 2a coleta $^{\text {a }}$ & 3a coleta & Médias \\
\cline { 2 - 5 } & \multicolumn{4}{c}{ mg por 100 g de alimento } \\
\hline $\mathbf{1}$ & $281,1 \pm 7,4^{\mathrm{D} \mathrm{b}}$ & $228,5 \pm 9,6^{\mathrm{CC}}$ & $323,8 \pm 9,8^{\mathrm{Ea}}$ & $277,8 \pm 42,1^{\mathrm{C}}$ \\
$\mathbf{2}$ & $429,7 \pm 3,0^{\mathrm{B} \mathrm{b}}$ & $516,7 \pm 14,2^{\mathrm{Aa}}$ & $394,2 \pm 16,9^{\mathrm{D}}$ & $446,8 \pm 55,7^{\mathrm{AB}}$ \\
$\mathbf{3}$ & $379,8 \pm 14,3^{\mathrm{Cb}}$ & $309,4 \pm 9,0^{\mathrm{B}}$ & $514,4 \pm 4,4^{\mathrm{Ca}}$ & $401,2 \pm 90,6^{\mathrm{BC}}$ \\
$\mathbf{4}$ & $485,5 \pm 28,4^{\mathrm{Ab}}$ & $532,6 \pm 20,9^{\mathrm{Ab}}$ & $696,6 \pm 16,6^{\mathrm{Aa}}$ & $571,6 \pm 97,1^{\mathrm{A}}$ \\
$\mathbf{5}$ & $109,5 \pm 6,1^{\mathrm{Eb}}$ & $122,7 \pm 4,0^{\mathrm{Eb}}$ & $549,9 \pm 7,6^{\mathrm{Ba}}$ & $260,7 \pm 217,0^{\mathrm{C}}$ \\
\hline
\end{tabular}

Valores expressos como média \pm desvio padrão seguidos por letras que indicam diferença estatística significativa em nível de 5\% pelo teste de Tukey; letras maiúsculas correspondem às diferenças estatísticas entre as médias das colunas e letras minúsculas às diferenças entre as médias das linhas

Durante as coletas, foi observado que algumas amostras de feijão apresentavam-se com pedaços de carne ou de produtos cárneos (amostras dos restaurantes 1 e 3 na $1^{\text {a }}$ coleta, 2 na $2^{\text {a }}$ coleta e de todos os restaurantes na $3^{\text {a }}$ coleta). Tal constatação não refletiu em diferenças significativas nos teores de sódio entre os restaurantes, mas as amostras não temperadas apresentaram valores significativamente inferiores nos teores de sódio entre as coletas (Tabela 2).

Dos resultados expostos na Tabela 3, observa-se que diferentes restaurantes apresentaram maiores teores de sódio na carne bovina em cada coleta, sendo inicialmente maior para a amostra do restaurante 4, seguido dos estabelecimentos 1 e 5 , que na média das coletas apresentaram os maiores teores de sódio na carne. $O$ restaurante 2 demonstrou valores bastante inferiores aos demais nos teores de sódio na carne bovina, apresentando apenas 13,2; 23,2 e 10,7\% do teor de sódio em comparação aos maiores valores encontrados dentre as carnes bovinas da $1^{\mathrm{a}}, 2^{\mathrm{a}}$ e $3^{\mathrm{a}}$ coletas, respectivamente. No entanto, na média das coletas, o restaurante 2 não diferiu significativamente do restaurante 3 .

Tabela 3. Teor de sódio na carne bovina assada oferecida pelos restaurantes comerciais do tipo self-service. Itaqui, RS, 2014

\begin{tabular}{|c|c|c|c|c|}
\hline \multirow[t]{2}{*}{ Restaurante } & 19 coleta & 2a coleta & 3a coleta & Médias \\
\hline & \multicolumn{4}{|c|}{ mg por $100 \mathrm{~g}$ de alimento } \\
\hline 1 & $753,2 \pm 25,2^{\mathrm{Cc}}$ & $935,5 \pm 23,0^{A b}$ & $1280,2 \pm 47,7^{\mathrm{B} \mathrm{a}}$ & $986,3 \pm 228,9^{\mathrm{AB}}$ \\
\hline 2 & $163,8 \pm 5,3^{\mathrm{Eb}}$ & $217,7 \pm 14,9^{\mathrm{Ca}}$ & $187,1 \pm 5,4^{\mathrm{Eb}}$ & $189,5 \pm 24,9^{D}$ \\
\hline 3 & $348,5 \pm 17,4^{D c}$ & $404,8 \pm 12,3^{\mathrm{B} \mathrm{b}}$ & $737,8 \pm 12,0^{\mathrm{Ca}}$ & $497,0 \pm 182,6^{C D}$ \\
\hline 4 & $1233,3 \pm 16,6 \mathrm{~A} \mathrm{a}$ & $409,3 \pm 21,4^{B C}$ & $636,3 \pm 9,7^{\mathrm{Db}}$ & $759,7 \pm 368,9^{\mathrm{BC}}$ \\
\hline 5 & $939,3 \pm 11,3^{\mathrm{B} \mathrm{b}}$ & $893,6 \pm 79,8^{A ~ b}$ & $1739,8 \pm 30,6$ А а & $1190,9 \pm 414,4^{A}$ \\
\hline
\end{tabular}

Valores expressos como média \pm desvio padrão seguidos por letras que indicam diferença estatística significativa em nível de $5 \%$ pelo teste de Tukey; letras maiúsculas correspondem às diferenças estatísticas entre as médias das colunas e letras minúsculas às diferenças entre as médias das linhas 
Para três dos restaurantes (1, 3 e 5$)$, os maiores teores de sódio ocorreram na $3^{a}$ coleta; já para os restaurantes 2 e 4 ocorreram na $2^{a}$ e $1^{a}$ coleta, respectivamente. Com exceção do restaurante 2 , os teores de sódio da carne bovina foram maiores do que nas amostras de arroz e feijão.

De acordo com a Figura 1, pode-se constatar a elevada contribuição em sódio das três preparações analisadas, que representam a base da refeição almoço, pela somatória das porções nas quantidades descritas no Guia Alimentar para a População Brasileira [23]. Vale ressaltar que os restaurantes comerciais não têm obrigação com limites para os teores de sódio dos alimentos ou do somatório das preparações. No entanto, utiliza-se como referência o Programa de Alimentação do Trabalhador (PAT) [24], que inclui a recomendação de 720 a $960 \mathrm{mg}$ de sódio para a refeição almoço, pois o programa estabelece parâmetros visando uma alimentação saudável.

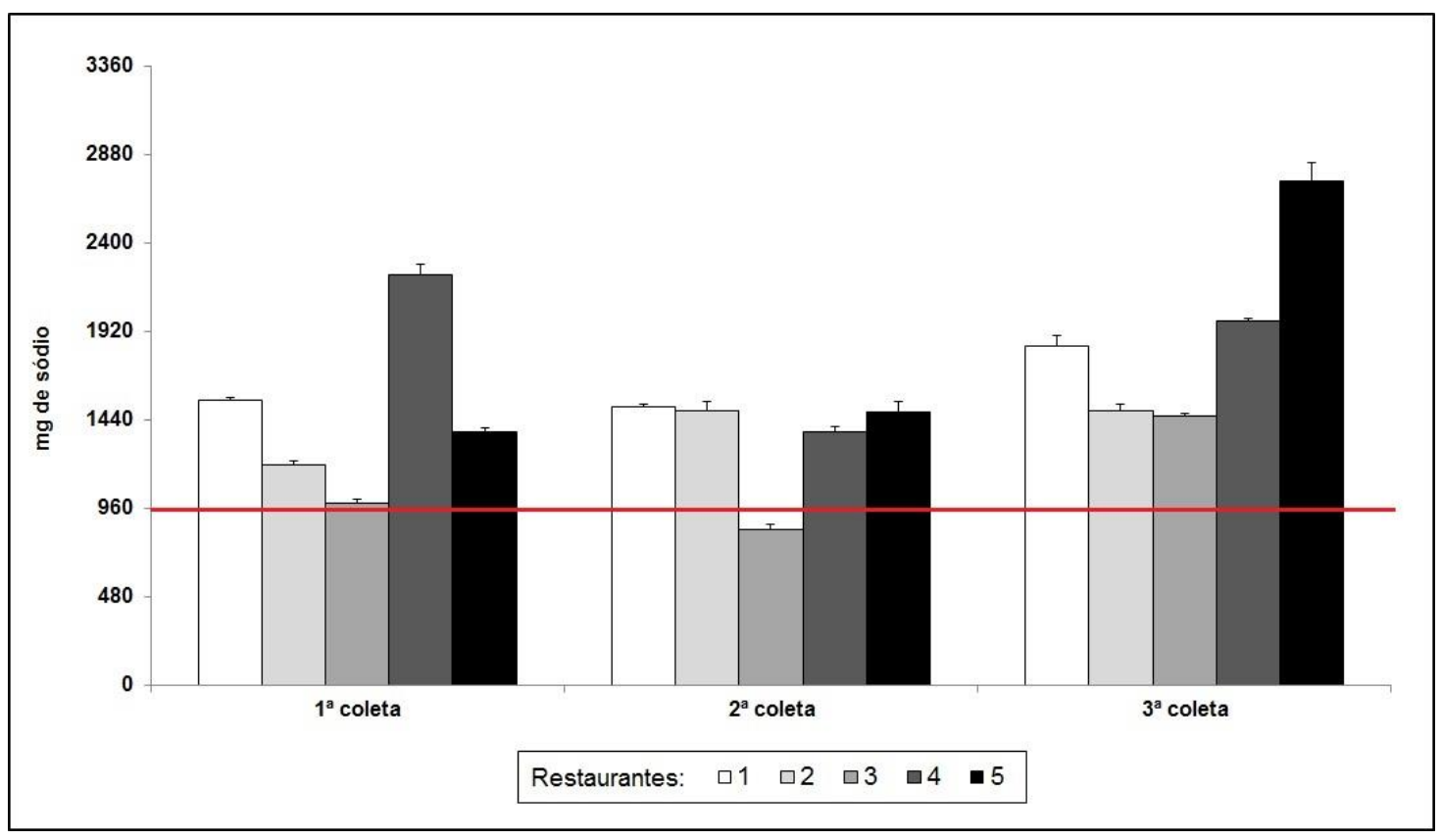

Figura 1. Teor de sódio na somatória das porções* de arroz branco, feijão em caldo e carne bovina assada oferecidas pelos restaurantes comerciais do tipo self-service comparadas à recomendação máxima do nutriente para a refeição**. Itaqui, RS, 2014

*Guia Alimentar para a População Brasileira [23]: $125 \mathrm{~g}$ de arroz, $86 \mathrm{~g}$ de feijão e $90 \mathrm{~g}$ de carne bovina

**Programa de Alimentação do Trabalhador [24]: 720 a 960 mg de sódio por almoço

$\mathrm{Na}$ Figura 1 fica bastante evidente que a somatória dos teores de sódio das três preparações analisadas supera a recomendação do PAT [24], com exceção do restaurante 3 , que não superou o limite de $960 \mathrm{mg}$ do mineral, porém apenas na $2^{\mathrm{a}}$ coleta. Em cada coleta, diferentes restaurantes apresentaram valores significativamente mais elevados na somatória das porções, sendo maior no restaurante 4 na $1^{\text {a }}$ coleta, nos restaurantes 1,2 e 5 na $2^{\text {a }}$ coleta e no restaurante 5 na $3^{\text {a }}$ coleta. Nas três coletas, $\mathrm{O}$ restaurante 3 apresentou significativamente menor somatória para o teor de sódio, embora na $3^{\text {a }}$ coleta não tenha diferido do restaurante 2 .

\section{DISCUSSÃO}

A técnica dietética [2] recomenda o uso de apenas $1 \%$ de sal no arroz, mas a clássica recomendação é de 1,5\% para o peso do cereal cru [25], que inclusive consta no modo de preparo da maioria das embalagens de produtos comerciais. Considerando 2,5 como fator térmico [26], os limites máximos ficam em aproximadamente 157 e 236 $\mathrm{mg} \%$ de sódio no arroz cozido para as 
recomendações de 1 e 1,5\%, respectivamente. No primeiro caso, todas as amostras superam o limite no uso de sal, enquanto que, apenas a amostra do restaurante 3 na $2^{\text {a }}$ coleta cumpre a clássica recomendação (Tabela 1).

Não há uma recomendação oficial para os teores de sódio ou sal no feijão, sendo que no preparo deste alimento também são utilizados diversos temperos e condimentos, normalmente com sal na composição. A recomendação dietética [2] é de 1\% de sal que, se considerado o valor de 3 como fator térmico [26] representa um limite de $131 \mathrm{mg} \%$ de sódio no feijão cozido. Desta forma, as amostras de feijão cozido do restaurante 5 estiveram ajustadas à recomendação, mas apenas na $1^{\mathrm{a}} \mathrm{e} 2^{\mathrm{a}}$ coleta (Tabela 2 ).

A necessidade de reduzir os teores de sódio no feijão foi constatada por Borjes et al. [11] que avaliaram sensorialmente o feijão de restaurantes comerciais com redução de sal em 50 e $75 \%$, a partir da substituição por uma especiaria (pimentado-reino branca moída) e três ervas aromáticas desidratadas (manjericão, manjerona e orégano). Esses autores observaram que o feijão com substituição do sal não atingiu bons índices de aceitação, com média em torno de $45 \%$.

No entanto, Patel et al. [27] demonstraram ser possível reduzir calorias, gordura, gordura saturada e sódio sem afetar a aceitação em 19 de 24 alimentos oferecidos no cardápio de restaurantes dos Estados Unidos. Especificamente para o teor de sódio, os autores mantiveram a aceitação após uma redução de $31 \%$ por porção e destacaram que essa redução pode ser efetiva na promoção da saúde para indivíduos que se alimentam frequentemente fora do lar.

Arroz e feijãoin natura são considerados alimentos de baixo teor de sódio, mesmo assim estão entre os alimentos que mais contribuem no consumo de sódio da população brasileira [28], provavelmente devido à representatividade desses alimentos na dieta do brasileiro, mas também ao uso excessivo de sal.

Como as demais amostras, a carne não tem uma recomendação oficial para adição de sal. A recomendação dietética [2] é de apenas $0,8 \%$ de sal que, se considerada uma variação de 0,6 a 0,9 no fator térmico [26], o teor de sódio na carne cozida deveria variar de 189 a $283 \mathrm{mg}^{\%}$. Assim, apenas as amostras de carne do restaurante 2 apresentaram teores de sódio inferiores ao limite superior da faixa estimada (Tabela 3).

Além de superar o limite de sódio recomendado pelo PAT [24], a contribuição em sódio das três preparações avaliadas supera o limite de ingestão diária recomendada pela OMS em duas coletas (restaurante 4 na $1^{\text {a }}$ coleta e restaurante 5 na $3^{a}$ coleta). Em outras dez situações, a somatória supera $1300 \mathrm{mg}$, ou seja, supre mais de $65 \%$ da quantidade diária de sódio recomendada pela OMS [9] (Figura 1).

Os expressivos teores de sódio nas preparações avaliadas acarretaram na elevação da estimativa para a refeição, conforme quantidades descritas no Guia Alimentar para a População Brasileira [23], que estabelece porções para a refeição almoço. Vale ressaltar que a somatória das porções não representa o total de sódio ingerido na refeição, pois apenas três preparações oferecidas no buffet dos restaurantes foram analisadas para o teor deste mineral. Normalmente, os consumidores incluem saladas e guarnições ao prato contendo sódio intrínseco e adicionado. Sal na mesa e condimentos à base de sal também contribuem para um elevado consumo de sódio ${ }^{[28]}$.

Kunert et al. ${ }^{[18]}$ pesquisaram as quantidades médias de sal e óleo adicionadas aos alimentos durante o preparo em restaurantes comerciais e também observaram que estes ultrapassam a recomendação diária per capita proposta pelo Guia Alimentar para a População Brasileira [23].

As variações constatadas nos teores de sódio entre as coletas das amostras dos mesmos restaurantes indicam que esses não possuem ou não seguem fichas técnicas. A Resolução No 380/2005 do Conselho Federal de Nutricionistas (CFN) [17], que dispõe sobre as áreas de atuação e atribuições do profissional nutricionista, compreende a elaboração de fichas técnicas em restaurantes para o controle dos processos de produção e de custos. A elaboração destas fichas técnicas requer bastante trabalho, mas é necessário, não apenas na padronização do sal e demais ingredientes, mas também para garantir a qualidade das refeições e prevenir prejuízos [29].

Entretanto, alguns trabalhos descrevem inadequações em refeições de Unidades de 
Alimentação e Nutrição (UAN), mesmo com supervisão de profissionais nutricionistas. Salas et al. [7] avaliaram teores de sódio em refeições almoço de uma UAN no município de Suzano, SP e também encontraram valores acima das recomendações do PAT, com valor médio de $2435 \mathrm{mg}$ em uma porção média (745 g) da refeição almoço. Duarte et al. [20] constataram que o almoço em uma UAN de um empresa privada não estava adequado nutricionalmente; a refeição excedia as recomendações estabelecidas pelo PAT para o valor energético total, macronutrientes, percentual proteico-calórico, fibras e sódio, que excedeu em até $150,15 \%$ a recomendação do PAT. Carneiro et al. [30] verificaram que o almoço de uma UAN cadastrada no PAT apresentou inadequações nos nutrientes analisados a partir das fichas técnicas, que no caso do sódio, a média semanal superou em $217 \%$ o limite máximo preconizado.

\section{CONCLUSÃO}

Os resultados demonstram que o sal é demasiadamente utilizado em preparações de arroz branco, feijão em caldo e carne assada oferecidas em cinco restaurantes comerciais da cidade de Itaqui, RS. Teores de sódio em conformidade com recomendações dietéticas foram encontrados apenas emduas amostras de feijão $(15 \%)$ e três de carne (20\%). Com base nas recomendações do PAT, a somatória das porçõesde três preparações da refeição almoço só não superou o limite máximo de sódio na $2^{a}$ coleta do restaurante 3 .

A variação do teor de sódio entre restaurantes e entre as amostras coletadas de um mesmo restaurante evidenciam a falta de padronização no uso de sal e a não utilização de fichas técnicas com a descrição da proporção de sal adequada às recomendações dietéticas para cada alimento.

\section{REFERÊNCIAS}

[1] Brasil.Ministério da Saúde. Secretaria de Atenção à Saúde. Departamento de Atenção Básica. Política nacional de alimentação e nutrição. 2 Ed. Brasília: Editora MS; 2007. 48 p.

[2] Domene SMA. Técnica Dietética: teoria e aplicações. Rio de Janeiro: Guanabara Koogan; 2011. 260 p.
[3] Schimidt MI, Duncan BB, Silva GA, Menezes AM, Monteiro CA, Barreto SM, et al. Doenças crônicas não transmissíveis no Brasil: carga e desafios atuais. Lancet. 2011;61-74.

[4] Sarno F, Claro RM, Levy RB, Bandoni DH, Ferreira SR, Monteiro CA. Estimativa de consumo de sódio pela população brasileira, 2002-2003. Rev Saúde Publica. 2009;43(2):219-25.

[5] Brow JI, Tzoulaki I, Candeias V, Elliott P. Salt intakes around the world: implications for public health. Int JEpidemiol. 2009;38(3):791-83.

[6] Spinelli MGN, Kawashima LM, Egashira EM. Análise de sódio em preparações habitualmente consumidas em restaurantes self service. Alim Nutr. 2011;22(1):55-61.

[7] Salas CKTS, Spinelli MGN, Kawashima LM, Ueda AM. Teores de sódio e lipídios em refeições almoço consumidas por trabalhadores de uma empresa do município de Suzano, SP. Rev Nutr. 2009;22(3):331-9.

[8] Sarno F, Claro RM, Levy RB, Bandoni DH, Monteiro CA. Estimativa de consumo de sódio pela população brasileira, 2008-2009. Rev SaúdePública. 2013;47(3):571-8.

[9] World Health Organization. Statement on management of hypertension.J Hypertesis. 2003;21(11):1983-92.

[10] Instituto Brasileiro de Geografia e Estatística. Pesquisa de orçamentos familiares 2008-2009: análise do consumo alimentar pessoal no Brasil[internet]. Rio de Janeiro: IBGE; 2011 [acesso em 14fev2017]. Disponível em: <http://www.ibge.gov.br/home/estatistica/popula cao/condicaodevida/pof/2008_2009_analise_cons umo/pofanalise_2008_2009.pdf>.

[11] Borjes LC, Techio SF, Oliveira MP. Análise sensorial de feijões de restaurantes comerciais com substituição do sal por ervas e especiarias. Nutrire: RevSocBrasAlim Nutr. 2011;36(3):15-26.

[12] Garcia RWD. Reflexos da globalização na cultura alimentar: considerações sobre as mudanças na alimentação urbana. Rev Nutr. 2003;16(4):48392. 
[13] Proença RPC, Sousa AA, Veiros MB, Hering B. Qualidade nutricional e sensorial na produção de refeições. Florianópolis: Ed. UFSC; 2008. 221 p.

[14] Magnée HM. Manual do self-service. São Paulo: Varela; 1996.242 p.

[15] Bezerra IN, Souza AM, Pereira RA, Sichieri R. Consumo de alimentos fora do domicílio no Brasil. Rev Saúde Pública. 2013;47(1Supl):200S-11S.

[16] Instituto Brasileiro de Geografia e Estatística. Cidades [internet]. Rio de Janeiro: IBGE; 2010 [acesso em 11 abr 2017]. Disponível em: <http://www.cidades.ibge.gov.br/painel/painel.ph p?lang $=\&$ codmun $=431060 \&$ search $=$ rio-grande-dosul\%7Citaqui $\% 7$ Cinfograficos:-dados-gerais-domunicipio>.

[17] Conselho Federal de Nutricionistas. Resolução 380/2005 - Dispõe sobre a definição das áreas de atuação do nutricionista e suas atribuições, estabelece parâmetros numéricos de referência, por área de atuação, e dá outras providências [internet]. Brasília; 2005. 45 p. [acesso em 11 abr2017]. Disponível

em:http://www.cfn.org.br/novosite/pdf/res/2005 /res380.pdf

[18] Kunert CS, Morais MP, Carvalho ACMS. Teores de sal e gordura nas preparações de restaurantes comerciais da cidade de Goiânia-GO. RevBrasPromoç Saúde. 2012;26(1):18-25.

[19] Spinelli MGN, Koga TT. Avaliação do consumo de sal em uma unidade de alimentação e nutrição. Nutrire: RevSocBrasAlim Nutr. 2007;32(2):15-27.

[20] Duarte MSL, Conceição LL, Castro LCV, Souza ECG. Qualidade do almoço de trabalhadores segundo o Programa de Alimentação do Trabalhador e o Índice de Qualidade da Refeição. Segur. Aliment. Nutr. 2015;22(1):654-61.

[21] Tedesco MJ, Gianello C, Bissani CA, Bohnen H, Volkweiss SJ. Análises de solo, plantas e outros materiais. $2^{\mathrm{a}}$ ed. Porto Alegre: Boletim Técnico do Departamento de Solos da Universidade Federal do Rio Grande do Sul; 1995. 174 p.
[22] Statistica: data analysis software system [programa de computador]. Versão 8.0. Tulsa: StatSoft, Inc.; 2007.

[23] Brasil. Ministério da Saúde. Secretaria de Atenção à Saúde. Departamento de Atenção Básica. Guia alimentar para a população brasileira: promovendo a alimentação saudável. Brasília: Editora MS; 2008. 210 p.

[24] Brasil. Portaria n. 193, de 05 de dezembro de 2006. Altera os parâmetros nutricionais do Programa de Alimentação do Trabalhador - PAT. Diário Oficial [da] República Federativa do Brasil, Brasília, DF, 7 dez. 2006. Seção 1, p. 123.

[25] GularteMA,MorasA, Prestes RB, SchirmerMA, Dias ARG, Elias MC. Efeito da proporção do sal de cozinha na cocção de arroz. In: 4. Congresso Brasileiro de Arroz Irrigado; 2005; Santa Maria. Santa Maria: Orium; 2005. p.349-51.

[26] Ornellas LH. Técnica Dietética: seleção e preparo de alimentos. $8^{a}$ ed. São Paulo: Atheneu; 2007. 276 p.

[27] Patel AA, Lopez NV, Lawless HT, Njike V, Beleche M, Katz DL. Reducing calories, fat, saturated fat, and sodium in restaurant menu items: effects on consumer acceptance. Obesity. 2016;24(12):2497-508.

[28] Souza AM, Souza BSN, Bezerra IN, Sichieri R. Impacto da redução do teor de sódio em alimentos processados no consumo de sódio no Brasil. Cad, Saúde Pública. 2016;32(2):e00064615.

[29] Souza LV, Marsi TCO. Importância da ficha técnica em UANs: produção e custos de preparações/refeições. J Health Sci Inst. 2015;33(3):248-53.

[30] Carneiro NS, Moura CMA, Souza SCC. Avaliação do almoço em uma unidade de alimentação e nutrição, segundo os critérios do programa de alimentação do trabalhador. Alim Nutr. 2013;24(3):361-5. 